\title{
A Digitally-Defined Analog Scheme to Aid Assessment of Food Colours
}

\author{
Adeyemo S. M. ${ }^{1, *}$, Popoola O. M. ${ }^{2}$ \\ ${ }^{1}$ Microbiology Department, Obafemi Awolowo University, Ile Ife, Nigeria \\ ${ }^{2}$ Kappa Biotechnologies and Laboratories, Trans Amusement Park, Ibadan, Nigeria
}

\begin{abstract}
Determination of food colour by sensory means could be subjective without instrumental assistance. This paper discusses a field comparator that can be constructed cheaply with local skills and materials for assisting sensory analysts in assessing food colours. For each food sample, a colour scheme can be defined in terms of the RGB digital combinations that are universally replicable but arranged into an analog scale. Its advantage is that sensory analysis is aided such that assessors essentially speak similar languages with respect to colour assessment. The scheme is suitable for use in field work or laboratories in developing countries where budgets are tight as the cost of the comparator is low, requiring only simple joinery and computing skills to construct. The range of colours for a particular assessment can be defined by any average computer user using the colour fill tool of a word processing package. Trials using the comparator with different colour disks are also reported. They show that untrained assessors reach close conclusions and standard deviations are reduced compared to similar but unaided assessors. While the scale selected for this report is 1 to 10 other scales may be selected and defined by individual workers in universally-defined tests like the hedonic scales.
\end{abstract}

Keywords Colour determination, Food colours, Comparator, Assessment, Scale, Subjectivity

\section{Introduction}

Food scientists, food technologists, other food commodity scientists, workers in food commerce as well as sensory evaluation panelists are often called upon to offer opinions on organoleptic properties of food. Methods for taste and aroma determinations by expert and non-experts have been well established to convert subjective human assessment to objective scientific parameters. Textural properties are also well instrumented. Much as there are instruments for measuring colours, in many cases in developing countries, judgment on colours are often left to subjective assessment. This is because many laboratories lack standard comparators or the right colour disks where comparators are available or are too expensive for use in field activities and laboratory organoleptic assessments.

The colour of food may need to be determined as a quality factor or in terms of hues of the same colour attached to a particular food, as they vary with varieties of a commodity. For example, it is common in food inspection to have to use colour as a measure of freshness in items like meat. On the other hand, the colours of fruits and vegetable products like carrot or tomato may vary widely in hues among varieties of

* Corresponding author:

adeyemostella@gmail.com (Adeyemo S. M.)

Published online at http://journal.sapub.org/microbiology

Copyright (C) 2015 Scientific \& Academic Publishing. All Rights Reserved the same commodity. With the scheme being described, once a fixed colour or hue of the same colour is selected, all other shades of the same colour can be defined relative to it. In addition, it is possible to define the colour outcome in mixtures of coloured foods using the assessor's own scale.

Foods acquire their colours through natural pigments mostly from plant materials, inorganic pigments, and synthetic coal-tar dyes (Bender, 1995). According to Gridgeman, 1997, assessors of taste must be able to use subjects who can recognize the following sweetness (sucrose-1.5\%); saltiness (sodium chloride-0.15\%); sourness (citric acid- $0.06 \%$ ) and bitterness-quinine sulphate- $0.005 \%$ ). It is hoped it will be possible to identify panelists who are equally sensitive to colours after selection using this scheme. Thus the scheme can be used to select sensitive trainers for determining hues and colours of food items, in addition to reducing the standard deviation of scores when untrained panelists are used.

Food quality is the quality characteristics of food that is acceptable to consumers. This includes external factors as appearance (size, shape, colour, gloss, and consistency), texture, and flavour; factors such as federal grade standards and internal (chemical, physical, microbial standard (Potter and Hotchkiss 1995).

Food quality in different countries is enacted by the Food Safety Act and enforced by such countries. There is also a need to monitor such in the market especially before they are processed; such food whose colour describe their quality are 
highly perishable. For example, meat, tomatoes, oranges, maize, palm oil carrot and so on. Members of the public complain to trading standards professionals, who submit complaint samples and also samples used to routinely monitor the food marketplace to public analysts (Sachin et al., 2015). Public analysts carry out scientific analysis on the samples to determine whether the quality is of sufficient standard.

There are many existing international quality institutes testing food products in order to indicate to all consumers which are higher quality products. The international quality institute Monde Selection is the oldest one in evaluating food quality. During the evaluation and analysis, products must meet the following selection criteria, required by the Institute: sensory analysis, bacteriological and chemical analysis, the nutrition and health claims, and the utilization notice. In short, the judgments are based on the following areas: colour, taste, health, convenience, labelling, packaging, environmental friendliness, methods and instrumentation (Sachin et al., 2015).

According to Alamu et al. (2015), food colour is very important in assaying the quality of coloured food product and proper processing method. He opined that hybrid variety and harvesting time were very important factors in rating sensory properties of boiled orange maize hybrids, and the sensory properties that determined the acceptance/likeness were found to be aroma, chewiness and taste. This information can also help researchers in choosing proper cooking methods to increase the retention of high levels of carotenoids in orange maize that can be delivered to consumers through nutrition education (Alamu et al.2015).

The aim of developing the scale is to achieve as good as consistent quality in the food product being produced, selected for use in the industries or the best method of processing and that which is compatible with the market for which the product is designed.

\section{Materials and Methods}

\section{(i) Development of Digitally-defined Colours.}

The rather technical part of this system is in describing each shade or hue of a colour or colour range in terms of RGB values. This can be done in any word processing or desktop publishing computer program and the scale printed on a transparent medium. The different shades of colour were produced in MS Word document using the fill-colour scheme. Each colour is defined in terms of Red; Green and blue on a scale of 255 (Table 1). Thus the different hues can be replicated anywhere by selecting the RGB combinations. The colours are then arranged on an analog scale of 1 to 10 in a circle. This is printed on a transparent sheet with a colour laser printer (Fig 1).
Table 1. RGB Values of Different Hues of Food Materials

\begin{tabular}{|c|c|c|c|}
\hline $\begin{array}{l}\text { COLOUR HUES } \\
\text { ON SCALE 1-10 }\end{array}$ & R VALUE & G VALUE & B VALUE \\
\hline \multicolumn{4}{|l|}{ Fresh Meat } \\
\hline 1 & 217 & 13 & 13 \\
\hline 2 & 223 & 19 & 19 \\
\hline 3 & 240 & 20 & 20 \\
\hline 4 & 240 & 40 & 40 \\
\hline 5 & 239 & 70 & 86 \\
\hline 6 & 243 & 79 & 79 \\
\hline 7 & 230 & 91 & 88 \\
\hline 8 & 234 & 92 & 95 \\
\hline 9 & 240 & 112 & 115 \\
\hline 10 & 247 & 139 & 133 \\
\hline \multicolumn{4}{|l|}{ Palm Oil } \\
\hline 1 & 251 & 64 & 5 \\
\hline 2 & 255 & 51 & 0 \\
\hline 3 & 252 & 60 & 4 \\
\hline 4 & 241 & 56 & 5 \\
\hline 5 & 238 & 60 & 4 \\
\hline 6 & 255 & 0 & 0 \\
\hline 7 & 183 & 0 & 10 \\
\hline 8 & 192 & 0 & 0 \\
\hline 9 & 193 & 2 & 8 \\
\hline 10 & 176 & 0 & 0 \\
\hline $\begin{array}{l}\text { COLOUR HUES } \\
\text { ON SCALE 1-10 }\end{array}$ & R VALUE & G VALUE & B VALUE \\
\hline \multicolumn{4}{|l|}{ Fresh Meat } \\
\hline 1 & 217 & 13 & 13 \\
\hline 2 & 223 & 19 & 19 \\
\hline 3 & 240 & 20 & 20 \\
\hline 4 & 240 & 40 & 40 \\
\hline 5 & 239 & 70 & 86 \\
\hline 6 & 243 & 79 & 79 \\
\hline 7 & 230 & 91 & 88 \\
\hline 8 & 234 & 92 & 95 \\
\hline 9 & 240 & 112 & 115 \\
\hline 10 & 247 & 139 & 133 \\
\hline \multicolumn{4}{|l|}{ Palm Oil } \\
\hline 1 & 251 & 64 & 5 \\
\hline 2 & 255 & 51 & 0 \\
\hline 3 & 252 & 60 & 4 \\
\hline 4 & 241 & 56 & 5 \\
\hline 5 & 238 & 60 & 4 \\
\hline 6 & 255 & 0 & 0 \\
\hline 7 & 183 & 0 & 10 \\
\hline 8 & 192 & 0 & 0 \\
\hline 9 & 193 & 2 & 8 \\
\hline 10 & 176 & 0 & 0 \\
\hline
\end{tabular}




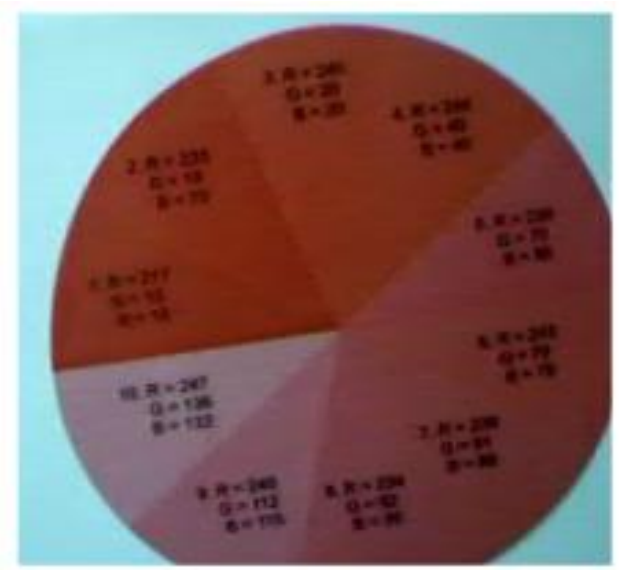

Figure 1. Construction of the Comparator Box

The disk described above is enclosed in the simple wooden comparator box of $5 \mathrm{~cm}$ x $19 \mathrm{~cm} \times 14 \mathrm{~cm}$ (Fig 2) that allows light to pass through two windows on it.

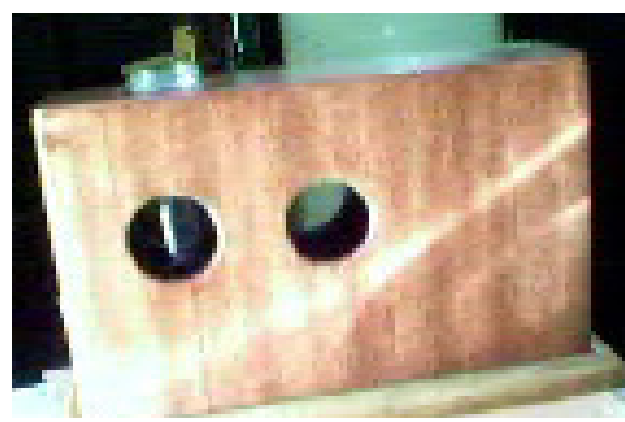

Figure 2. Wooden Comparator Box

Into a slit above one of the windows is slid the requisite colour disk for the product to be assessed and coming into view through the circular window. The other window contains a colourless sample bottle used as a cuvette for the Food sample, which may be solid or liquid.

To measure the colour of the food sample, it is placed in the cuvette and the disk corresponding to its main colour is slipped into the slot on the wooden box. The disk is rotated in its slot and the box viewed against white light. The disk is rotated slowly until a colour that matches is reached. The number of the colour is noted and its RGB definition recorded. So far disks containing different colours of red meat, yellowness of Carrot and yellow-ripening fruits and redness of tomato and red-ripening fruits have been defined. Other colours shades are being defined in this on-going project.

\section{(II) Field Trials Using the Comparator}

Trials were made with the system described above with different food items by untrained assessors. In all cases, samples of the food were cut in $1 \mathrm{~cm}$ cubes and placed in the cuvette (Sample bottle). The colour of the food items were matched against the colours on the disk in the field comparator as it is being rotated. A similar exercise was carried out on another set of assessors without the aid of the comparator. The standard deviations in the assessments were calculated. The standard deviation for the respondents without the comparator was high while that of the respondents with the colour aid was small.

Table 2.

\begin{tabular}{|c|c|c|c|}
\hline $\begin{array}{l}\text { Respondents' Number } \\
\text { (Without Colour Aid) }\end{array}$ & Scores & $\begin{array}{c}\text { Respondents' } \\
\text { Number (With } \\
\text { Colour Aid) }\end{array}$ & Scores \\
\hline 1 & $* 4$ & 1 & 6 \\
\hline 2 & 6 & 2 & 5 \\
\hline 3 & 8 & 3 & 6 \\
\hline 4 & 7 & 4 & 6 \\
\hline 5 & 8 & 5 & 6 \\
\hline 6 & 8 & 6 & 6 \\
\hline 7 & 3 & 7 & 6 \\
\hline 8 & 5 & 8 & 7 \\
\hline 9 & 5 & 9 & 6 \\
\hline 10 & 6 & 10 & 6 \\
\hline 11 & 4 & 11 & 5 \\
\hline 12 & 6 & 12 & 6 \\
\hline 13 & 5 & 1 & 6 \\
\hline 14 & 6 & 14 & 6 \\
\hline 15 & 4 & 15 & 7 \\
\hline 16 & 7 & 16 & 6 \\
\hline 17 & 5 & 17 & 6 \\
\hline 18 & 9 & 18 & 7 \\
\hline 19 & 3 & 19 & 7 \\
\hline 20 & 4 & 20 & 7 \\
\hline Mean $=$ & 5.65 & Mean & 6.15 \\
\hline Standard deviation & 8 & Standard deviation & 6 \\
\hline
\end{tabular}

*Figures obtained are means of replicate determinations

\section{Result}

The statistical analysis showed that for the samples that were observed without the colour aid, the standard deviation was high (8), the mean was 5.65 for these respondents; the dispersion was high also. The higher the variation from the mean, the lower the consistency. But for samples that were observed with the colour aid, the standard deviation was small; the mean was 6.15 while the standard deviation was 6 . It was observed that the dispersion was small. The lower the variation from the mean, the higher the consistency. Consistency in the colour of particular food sample is a pointer or index of quality. Quality means conformity to standards which meets consumer and customer evaluation of a desired product with no variation or when the variation is reduced to near zero level.

\section{Discussion}

Sensory evaluation of aspects of food quality is a human exercise. Even though choice of types and numbers of 
panelists with statistical analysis reduces this subjectivity, this comparator is set to further reduce subjectivity in colour determination of food materials. Similarly, food inspectors in the field should find this comparator handy and useful in routine work. For meat inspection, in a situation where fresh blood is poured on the previous day's meat to make it look fresh, a cut from the inside chunk of meat and use of this comparator to measure its colour at the core will reveal the true status of the meat. A trial of the methods has revealed that biases from different panelists have been narrowed down.

According to Gridgeman (1992), panelists have different criteria they must fulfill before they are made assessors for taste determination. Since we do not have such for colours, the above method can be used and serve as a guideline for determining colour in a controlled manner.

With the colour aid, the variation was lower and there was greater consistency compared to the values obtained without the colour aid.

Food quality is an important food processing and manufacturing requirement, because food consumers are susceptible to any form of contamination that may occur during the manufacturing process. Many consumers also rely on manufacturing and processing standards particularly to know what ingredients are present, due to dietary, nutritional requirements. Besides ingredient quality, there are also requirements for selection of raw materials from the field or market. It is important to ensure that the food processing environment is as clean as possible in order to produce the safest possible food for the consumer. Food quality also deals with product traceability, (e.g appearance, ingredient, and packaging suppliers) and colours, which is the first thing you notice on sighting any food sample (Alamu et al. 2015, Sachin et al., 2015).

\section{Conclusions}

Local instrumentation should be encouraged, particularly for field workers who may be operating far from base or even in laboratories where costs have limited the use of more precise instrumentation. Many quality parameters of biological materials such as colours, appearance, hue, pigmentation, taste, aroma, flavor, nutritional and organoleptic attributes change within short times and in the absence of sophisticated equipment; sufficient information for knowing their colours can be obtained this way. Hence, the instrument was developed for use in environment that lacks the access to such equipments.

\section{ACKNOWLEDGMENTS}

The authors wish to appreciate the contributions of the Management and Staff of Kappa Biotechnologies, Bodija, Ibadan for their contributions towards the practical aspect of the work.

\section{REFERECES}

[1] Bender, A.E. (1995). Dictionary of Nutrition and Food Technology. $6^{\text {th }}$ Edition Butterworth \&CO. (Publishers) Ltd.

[2] Gridgeman, N.T (1997). Taste Panel: Sensory Assessment in Quality Control in Quality control in Food industry Ed. Herschdoerfer, S.M. 3rd Edition. Academic Press London New York

[3] Alamu, O.E., Bussie, M.D., Abebe, M. and Olaofe, O. (2015). Effects Of Husk And Harvesting Time On Provitamin A Activity And Sensory Properties Of Boiled Fresh Orange Maize Hybrids. Elsevier Journal of Food Quality ISSN 1745-4557.2015 Wiley Periodicals, Inc.

[4] Sachin, K., Sonawane, M.B., Bagul, J., LeBlanc, G. and Shalini, S. A. (2015). Nutritional, functional, thermal and structural characteristics of Citrullus lanatus and Limonia acidissima seed flours. "Sensing and Instrumentation for Food Quality and Safety". Springer Journal of Food Measurement and Characterization, 23:1-8.

[5] Potter, Norman N. and Joseph H. Hotchkiss (1995). Food Science. 5th Edition. New York: Chapman \& Hall. pp. 90-112. 